\title{
Differentiating Birds and Animals using Deep Learning Neural Network with Image Processing Approach
}

\author{
K Pandiaraj \\ Assistant Professor \\ Department of Electronics and \\ Communication Engineering \\ Kalasalingam Academy of Research \\ and Education \\ Krishnan koil,Tamilnadu
}

\author{
P Sivakumar \\ Professor \\ Department of Electronics and \\ Communication Engineering \\ Kalasalingam Academy of Research \\ and Education \\ Krishnan koil,Tamilnadu
}

\author{
V Nandhini ,S Parkavi \\ Department of Electronics and \\ Communication Engineering \\ Kalasalingam Academy of Research \\ and Education \\ Krishnan koil,Tamilnadu
}

\begin{abstract}
In farms we can see that the birds and animals destroying the crops. The movement of birds and animals cannot be controlled by any method. We can only drive away them. To drive away them, humans are used. To reduce the human effort we have introduced a method using image processing. In this method, the real time images are given as input and sound will be derived as output. The image given as input is compared with the trained images and classified into birds and animals. After the identification, birds can be driven away by using cracker sound and animals can be driven away by using a human sound.
\end{abstract}

\section{INTRODUCTION}

In today's world automation plays an important role. Computer vision is a part of automation. Computer vision can be used in many discipline of automation; some of them are remote sensing, medical field, machine vision, and robotics. Computer vision can be applied for attendance management, surveillance purpose, and also in agricultural field. In agricultural field we can use computer vision for detecting diseases by examining the fruits and leaves, and it can be used in quality control of the agricultural products. In many agricultural fields we can see animal threat as a major problem. According to the reports of Himachal kisan shaba, every year farmers of Himachal Pradesh faces the loss of four hundred crore to five hundred crore. Many farmers started using electrical fence to avoid the movement of animals in agricultural land but the electrical fence kill the animals which is an offensive act. So the techniques which are used now is not that efficient. So we proposed a method to reduce the loss in farm by driving away the birds and animals by using sound. To differentiate bird and animal, first we are converting colour image to gray scale image, size will be corrected and blur in the image is removed by adding a filter. Then the image is segmented into $256 \times 256$ pixels. Then the features of the image such as contrast, correlation, energy, homogeneity, mean, standard deviation, entropy, RMS, Variance, smoothness, Kurtosis, Skewness is extracted using feature extraction and added into data sheet. $\mathrm{CNN}$ classifier is used for differentiating bird and animal. After the classification relevant sound will be generated to drive away birds and animals.

\section{RELATED WORKS}

Andr'eia Marini, Jacques Facon, Alessandro L. Koerich "Bird Species Classification Based on Color Features" ( 2013 IEEE International Conference on Systems, Man, and Cybernetics) in this paper for bird species classification they have presented a novel approach. Classification can we carried out from unconstrained images based on colour feature extraction. Which means birds appeared in different size, pose and angle of view can be identified. In this approach first colour segmentation algorithm is applied to remove the background elements. The second step is to split the image into component plane and normalised colour histograms are computed. Histogram bin values are used to distinguish number of birds. Then the experimental results are added to the datasheet. $75 \%$ segmentation value and $90 \%$ bird species classification is correct.

Baowen Qiao, Zuofeng Zhou, Hongtao Yang, Jianzhong Cao, "Bird Species Recognition based on SVM Classifier and Decision Tree", (2017 First International Conference on electronics instrumentation \& Information System(EIIS)), in this paper to classify the birds they have used the ratio between the distance of eye and tip of the beak. The received feature will be added to SVM frame work and multi-scale decision tree.

Slavomir Matuska, Robert Hudec, Miroslav Benco, Patrik Kamencay, Martina Zachariasova, "A Novel System for Automatic Detection and Classification of Animal", (2014 IEEE conference, Zilina, Slovakia). They have proposed a method in this paper to find the area of movement of the wild animals. This process is fully dependent on watching device. A $360^{\circ}$ surveillance system is connected to the server. In server we will have a system called automatic system for animal recognition which will recognize and classify the animal.

Lingzi Duan, Fusheng Yu, Li Zhan “An Improved Fuzzy C-means Clustering Algorithm”, (2016 12th International Conference on Natural Computation, Fuzzy Systems and Knowledge Discovery (ICNC-FSKD)). In this paper, to reduce the sensitivity of algorithm, clustering pattern is modified. First the clustering is done using fuzzy 
c-mean algorithm then the number of properties is identified. If properties of a cluster is more than one then the cluster is divided into two more clusters, with which we can find highly accurate classification.

\section{EXISTING MEYHOD}

To drive away birds and animals high human resource is necessary.

Disadvantage of existing method

waste of human resource

$>$ loss of crops in field

\section{PROPOSED METHOD}

Computer vision is used in many disciplines, we are introducing it into agriculture. When a colour image is given as input to the system it will differentiate it into bird and animal. To differentiate it as birds and animals the deep learning neural network with image processing approach is used. After differentiating birds and animals, sound will be played to drive away the bird or animal.

Advantage of the proposed method

- less human interaction

- Highly accurate due to Deep Neural network classifier.

- High sensitivity due to Genetic feature extraction

* Working

The process consist of

- Preprocessing

- Segmentation

- Feature extraction

- Classification

- Performance parameters calculation

\section{A. Prepocessing}

A image can be of any colour, size and it may be with noise. To recover a proper image we have to convert the colour image into gray scale image. Next we are resizing the image, image resizing is important because it makes the comparison process much accurate. To remove the noise we are using Gabor filter. Gabor filters are called as linear filters. By multiplying harmonic function with Gaussian function, the impulse response is defined. In various image processing techniques such as texture segmentation, iris pattern description and tumor detection, the response of Gabor filter is used successfully. The main advantage of this filter is that they satisfy the minimum space-bandwidth product per the uncertainty principle. Hence they provide simultaneous optimal resolution in both the space and spatial frequency domains.

\section{B. Segmentation}

For the process of segmenting images by pixel classification method, FCM clustering method is used. When the Fuzzy C-Means technique is used for brain tumor segmentation, a bunch of classes are obtained. According to its attributes (i.e. intensity, texture, etc.), every pixel is given membership value. The similarity degrees between the data value at a specific location and the typical data value, or centroid, of its class, are reflected by the fuzzy membership functions, which constrained to be between 0 and 1. The data value at that location is closed to the centroid of the class indicated by the membership value which is near to one. The concept of fuzzy membership is used by the Fuzzy C-Means algorithm. The algorithm contains following steps.

- Initialize u with random values between zero and one.

- Calculate objective function F

- Calculate centroids of the clusters $\mathrm{Cj}$

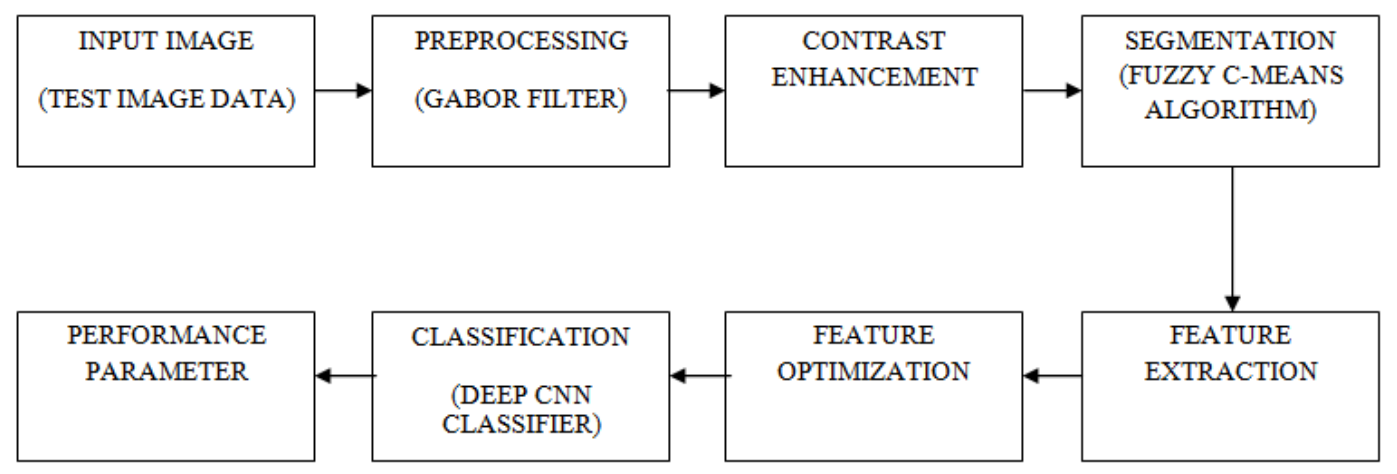

Fig 1:- Block Diagram

\section{Feature Extraction}

In region description Color, texture and shape are normally used. Texture is one of the significant feature to recognize and classify the object. For describing the texture, energy, contrast, Homogeneity, entropy and dissimilarity are used. To detect the intensity variation, the texture feature uses the GLCM algorithm.
Contrast: Contrast is used to detect the contrast between a pixel and its neighborhood value and it is computed by this equation

$\operatorname{CONTRAST}=\Sigma_{\mathrm{i}, \mathrm{j}}|\mathrm{i}-\mathrm{j}|^{2} \mathrm{p}(\mathrm{i}-\mathrm{j})$ 
Energy: The energy value is set to 1 for constant image. The sum of squared elements is returned, it is calculated using

$\operatorname{ENERGY}=\Sigma_{\mathrm{i}, \mathrm{j}} \mathrm{p}(\mathrm{i}, \mathrm{j})^{2}$

Homogeneity: The homogeneity is used to detect the closeness of the distribution of elements. It ranges from 0 to 1.

HOMOGENEITY $=\sum_{\mathrm{i}, \mathrm{j}} \frac{p(i, j)}{1+|i-j|}$

Correlation: Correlation is used to detect a pixel to its neighbor value. For attaining perfect positive and negative correlation the value ranges from-1 to 1 .

\section{Classification}

Convolutional Neural Network (CNN) is used for learning how to segment images. CNN extracts features directly from pixel images with minimal preprocessing. The network we use is LinkNet. It is a light deep neural network architecture designed for performing semantic segmentation.

\section{RESULTS}

\section{Input Image}

The image given as input is,

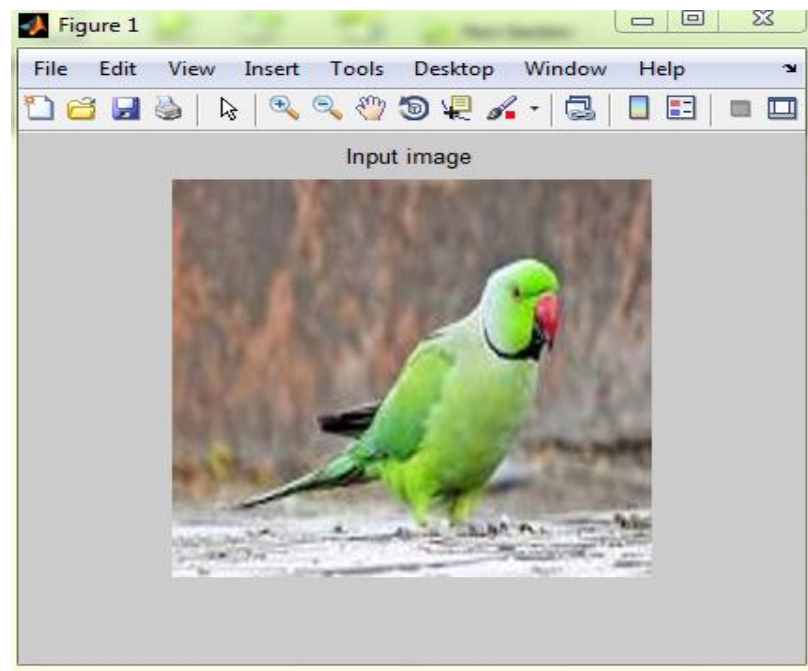

Fig 2:- Input image

\section{Pre Processing Result}

The input image is of any colour, size and it may contain noise. To get a proper image, the image must undergo preprocessing technique. The result of preprocessing technique is,
ISSN No:-2456-2165

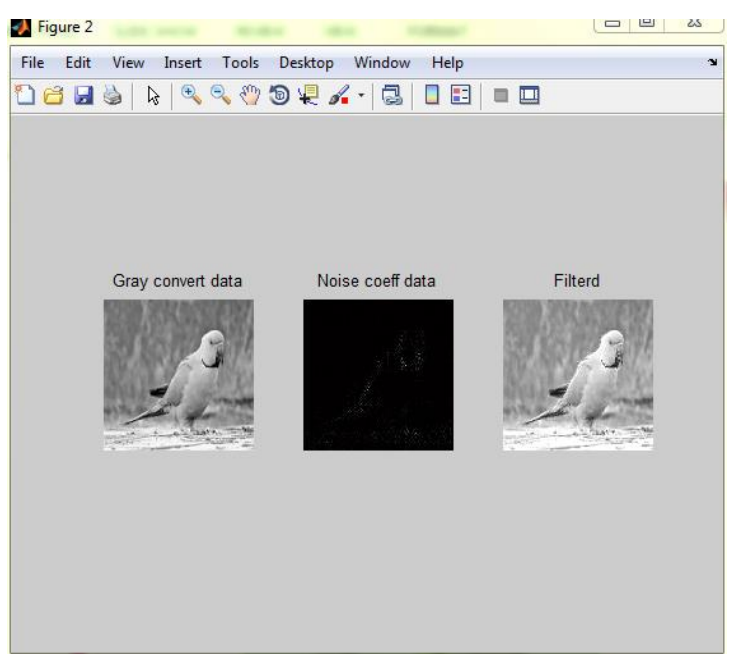

Fig 3:- Preprocessing result

\section{Enhanced Image}

Image enhancement technique is used to increase the quality of the image. The output of the enhanced input image is,

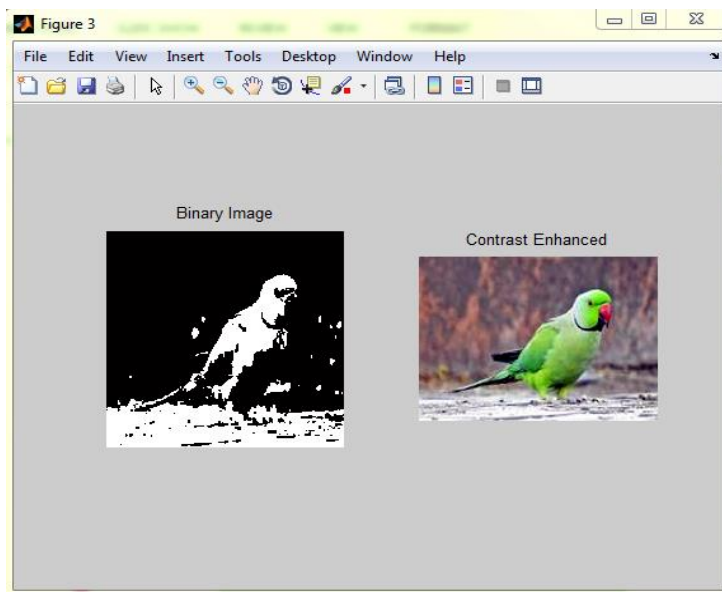

Fig 4:- Enhancement image

\section{Morphological Features}

Morphological image processing is used to remove the imperfections of the input image. The output of the image is,

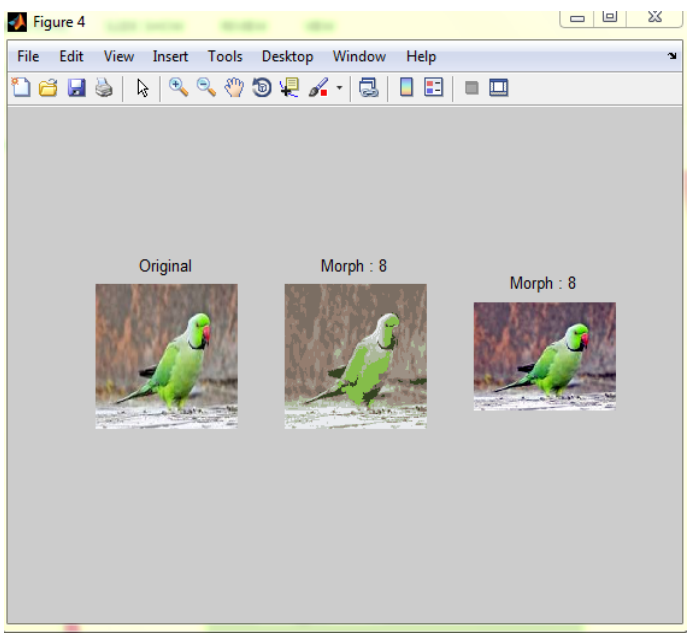

Fig 5:- Morphological feature 


\section{Clustering Result}

Clustering technique is used to partition the image sets into similar groups. The output of the clustered image is,

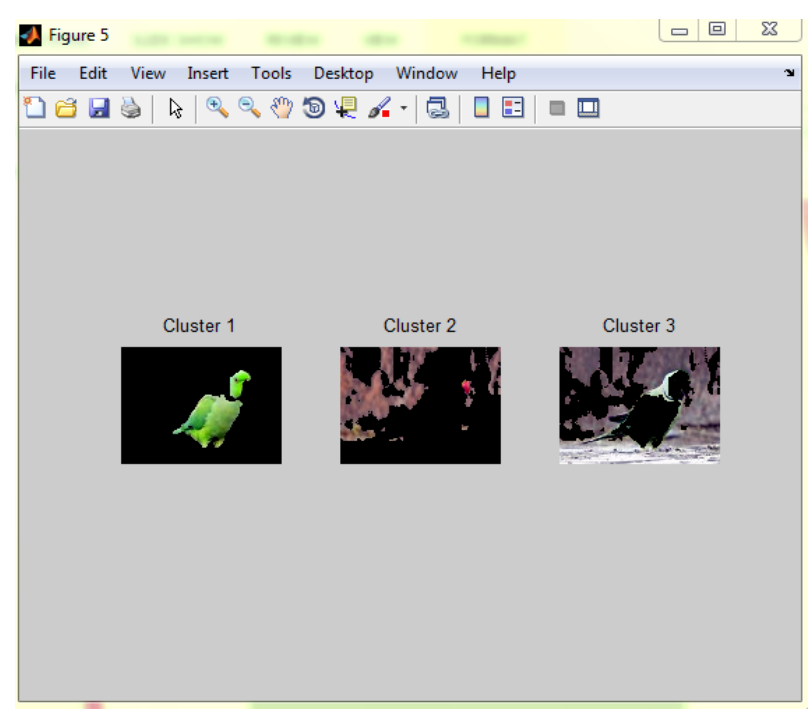

Fig 6:- Clustering result

\section{Segmented Image}

Image segmentation technique is the process of allocating label to each pixel. Pixel with same label share certain characteristics. Here we have used FCM algorithm for segmenting images.The output of the segmented input image is

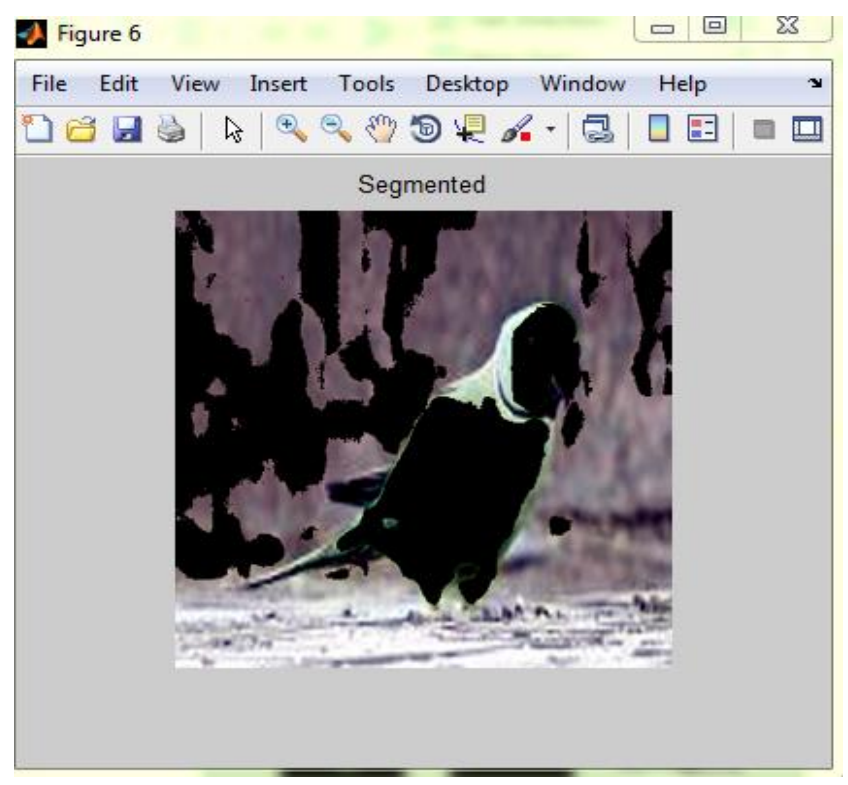

Fig.7:- Segmented image

\section{Feature Extraction Results}

Feature extraction is the process of extracting colour, texture and shape of an image. The output of feature extraction is

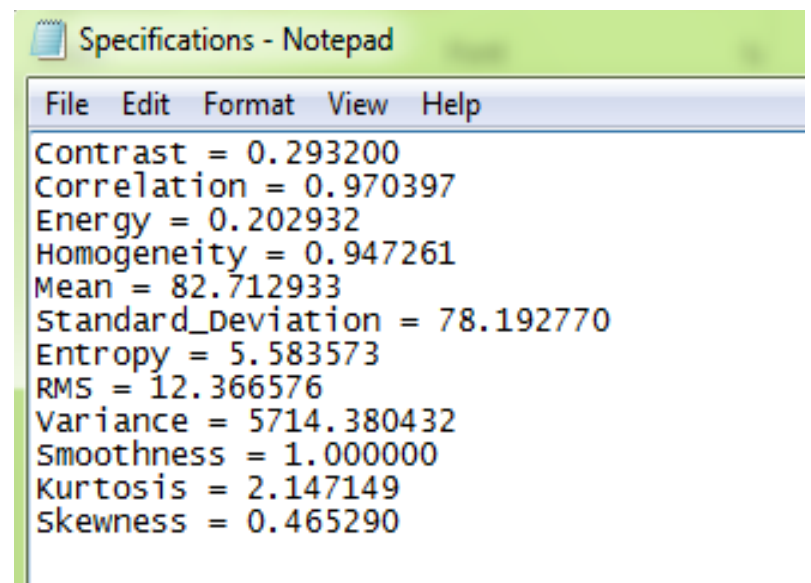

Fig 8:- Feature Extraction Result

\section{CONCLUSION}

An image processing technique is designed to drive away birds and animals in the field. This method has high accuracy because of deep neural network classifier. It is highly sensitive due to genetic feature extraction approach. Therefore this method is easy to implement and used to help the farmers in an effective way.

\section{REFERENCES}

[1]. Andreia Marini, Jacques Facon and Alessandro L. Koerich, Pontifical Catholic University of Paran'a (PUCPR), "Bird Species Classification Based on Color Features", proceeding of IEEE International Conference on Systems, Man, and Cybernetics ,2013.

[2]. Lingzi Duan, Fusheng Yu, Li Zhan, School of Mathematical Sciences, Beijing Normal University, "An Improved Fuzzy C-means Clustering Algorithm", proceeding of 12th International Conference on Natural Computation, Fuzzy Systems and Knowledge Discovery (ICNC-FSKD), 2016.

[3]. Baowen Qiao,Zuofeng Zhou, Hongtao Yang, Jianzhong Cao, 1Shaanxi normal university, "Bird Species Recognition based on SVM Classifier and Decision Tree", proceeding of first International Conference on electronics instrumentation \& Information System(EIIS), 2017.

[4]. Slavomir Matuska, Robert Hudec, Miroslav Benco, PatrikKamencay, Martina Zachariasova, Department of Telecommunication and Multimedia University of Zilina, "A Novel System for Automatic Detection and Classification of Animal", proceeding of IEEE conference,Zilina, Slovakia, 2014.

[5]. Chenggang Zhen, Xin Zhang, Xinjing Mao, Information and Network Management Center of North China Electric Power University, Baoding, "Research on Image Processing Based on Genetic Algorithm for Furnace Flame", proceeding of IEEE ISIE 2006, Montreal, Quebec, Canada, 2006. 
[6]. P Raghu Veera Chowdary, M Nagendra Babu, Thadigotla Venkata Subbareddy, Bommepalli Madhava Reddy, V Elamaran, Department of ECE, School of EEE,SASTRA University,Thanjavur, Tamilnadu, India, "Image Processing Algorithms for Gesture Recognition using MATLAB”, proceeding of IEEE International Conference on Advanced Communication Control and Computing Technologies (ICACCCT), 2014.

[7]. Vasanth Philomin, Ramani Duraiswami, and Larry Davis, Computer Vision Laboratory, Institute for Advanced Computer Studies University of Maryland, College Park, "Pedestrian tracking from a moving vehicle", proceedings of IEEE Intelligent Vehicles Symposium, 2000. 